Published in R. Luo (ed.), Encyclopedia of Color Science and Technology, doi DOI 10.1007/978-3-64227851-8_17-1

\title{
Chromatic Contrast Sensitivity
}

Christoph Witzel and Karl Gegenfurtner Department of Psychology, Giessen University, Giessen, Germany

\section{Definition}

Chromatic contrast refers to the occurrence of differences in chromaticity (saturated, huefull color) in a visual percept (scene, image, stimu- lus). It may consist in differences across space (spatial chromatic contrast) or in changes of chromaticity across time (temporal chromatic contrast). The term chromatic contrast is used in opposition to achromatic contrast, where differ- ences only occur in luminance (gray level). For example, whereas a black-and-white photo only contains achromatic contrasts, a color photo also contains chromatic contrast. While chromatic and color contrast refer to the same visual phe- nomenon, the term "chromatic contrast" empha- sizes research on chromatic contrast sensitivity.

\section{Conceptual Clarifications}

Almost every phenomenon in color vision involves contrasts between colors. This is partic- ularly true since colors are not perceived abso- lutely, but relative to other colors. In fact, contrasts between colors affect the appearance of the single colors. Still, the term chromatic contrast has been associated with certain aspects in the perception of color differences rather than others (for review, see [1]). In order to understand the particular connotations of chromatic contrast, it is useful to clarify the relationship of this term to achromatic and isochromatic contrast; the dis- tinction between spatial and temporal chromatic contrast; differences in connotation to color con- trast and color edges; the difference between color detection, color discrimination, and chro- matic contrast sensitivity; and the relationship between chromatic contrast sensitivity, spatial resolution, and chromatic acuity.

\section{Achromatic Contrast and Isochromatic Images}

Since human color vision involves an achromatic luminance dimension and two chromatic dimen- sions, visual contrasts may occur in terms of luminance or chromaticity. Hence, the term chro- matic contrast must be understood as the comple- ment of achromatic contrast [1]. Achromatic contrast refers to differences in luminance, which are perceived as lightness differences. Spatial achromatic contrasts may be illustrated by gray-scale images, such as black-and-white photos. Unlike achromatic contrast, chromatic 
contrast involves differences in chromaticity, which are differences along one or both of the chromatic dimensions as opposed to the achro- matic dimension.

Purely achromatic contrasts may occur in a chromatic image, for example, because the paper of a black-and-white photo yellowed over time or because the gray-scale image was printed on color paper. However, since such images only contain lightness differences, they are void of chromatic contrast [7]. Such an image (or stimulus) is called isochromatic because it is equal ("iso") in chromaticity [14].

\section{Spatial and Temporal Contrast}

Like any visual contrast, chromatic contrast may occur through variations across space or across time [1]. On the one hand, spatial chromatic contrast consists in the simultaneous occurrence of differ- ences in chromaticity at different locations in space. On the other hand, temporal chromatic contrast refers to changes in chromaticity over time. In both cases, the strength of the contrast is defined as the size of the difference in chromaticity.

However, the perception of contrast strongly depends on the transition between the two chro- maticities. Transitions can be rather gradual or abrupt. For example the boundary between a red shirt and a blue trouser provides an abrupt transi- tion between red and blue, while the transitions between the colors of a rainbow are more gradual. The temporal transition between the colors of a traffic light is abrupt, while the change of the chromaticity of sunlight during the day is very slow and gradual. Such transitions may be assessed by the spatial and temporal frequency of the color transition, respectively. The ability to perceive chromatic contrast depends on these spatial [7] and temporal frequencies [5].

\section{Color Contrast and Color Edges}

Like chromatic contrast, the term color contrast refers to the occurrence of differences in chroma- ticity, and both terms are often used interchange- ably. However, these terms highlight different aspects due to their usage in different research domains. On the one hand, the term chromatic contrast mostly (but not exclusively) refers to research on contrast sensitivity and its relation- ship to spatial and temporal frequency [1]. For this reason, the term chromatic contrast focuses on the distribution of color differences across space and time and the ability of the observer to detect these differences (contrast sensitivity). On the other hand, the term color contrast is predom- inantly used in the context of research on how the identity of a color is affected by its chromatic surround. For this reason, this term is associated with chromatic induction that is the effect of color contrasts on color appearance (the subjec- tive impression of color). In this context, lightness contrast is the gray-scale complement of color contrast.

Chromatic edges (or chromatic edge con- trast) are a particular kind of spatial chromatic contrast. Edge contrasts are related to the visual environment (scenes) because they are 
delimiting objects or other fundamental features of a scene such as shadows or highlights. As a result, while the term chromatic contrast focuses on the sensi- tivity to color contrasts in general, the notion of chromatic edges highlights the relationship between the beholder and their visual environ- ment and is rather used in the context of scene statistics (e.g., [3]).

\section{Detection, Discrimination, and Contrast Sensitivity}

Color detection, color discrimination, and con- trast sensitivity all refer in some way to the ability to see differences in chromaticity and hence in chromatic contrast. At the same time, they involve different experimental paradigms.

Color detection consists in the discrimination of one color (test) from the neutral (i.e., adapting) background. Chromatic sensitivity, the ability to see colors, is measured as the minimum differ- ence in chromaticity between test color and back- ground that can be detected.

Color discrimination consists in the discrim- ination between two different colors (test and comparison) shown on the adapting background. The ability to discriminate colors (sensitivity to color differences) is determined through the min- imum difference between the colors that can be discriminated on a given background. Color detection may be conceived as a special case of color discrimination, in which the test color is identical with the background. In general, the terms color detection, color discrimination, and color sensitivity typically refer to differences in chromaticity independent of spatial or temporal frequency.

Chromatic contrast sensitivity refers to the general ability to detect a contrast between colors. This ability depends on the temporal or spatial frequency with which color differences occur. As a result, contrast sensitivity concerns the detection of contrasts given a certain temporal or spatial distribution of these contrasts.

While color detection and discrimination are measured with single presentations of test and comparison colors, contrast sensitivity is mea- sured with periodical changes in chromaticity, mostly involving two opponent chromaticities (see section "Method"). Nevertheless, color detection and discrimination may be understood as special cases of chromatic contrast sensitivity. Chromatic contrast sensitivity converges to a maximum at low spatial and temporal frequen- cies and is zero above an upper boundary (chromatic acuity) of spatial frequencies (see section "Chromatic Contrast Sensitivity Func- tions"). By using sufficiently large color patches ( $>1$ - visual angle) and presenting them for a sufficiently long time ( $>200 \mathrm{~ms})$, contrast sensi- tivity will be at maximum, independent of addi- tional high spatial frequency (edges of stimuli) and high temporal frequency components (abrupt stimulus onset). In this way, color detection and discrimination may be measured (to a large extent) independent of spatial and temporal frequencies and reflect maximal chromatic contrast sensitivity in terms of spatial and temporal frequency. 


\section{Spatial Resolution and Chromatic Acuity}

Spatial resolution refers to the maximal spatial frequency that can still be seen, such as the minimum size of letters that can still be identi- fied. Because of the tight relationship between spatial frequency and contrast sensitivity, con- trast is also the complement of spatial resolution and acuity. For example, the smallest letters that can still be read when printed in black on a white paper may not be identified when printed in light gray instead of black, because they have lower contrast than the black letters. Hence, the spatial resolution depends on the contrast. This is also true for the spatial resolution of color vision. It can only be determined relative to a given con- trast: the thinnest color line that may be detected with a high chromatic contrast will be invisible with a lower contrast.

Visual acuity is a particular case of spatial resolution, which corresponds to the highest spa- tial frequency that can be detected at maximum contrast [1]. It can only be determined relative to a given contrast, such as the black letters on a white background. Analogically, chromatic acu- ity corresponds to the highest spatial frequency that is still visible when presented with maximum chromatic contrast (e.g., [7]).

\section{Method}

In order to separate luminance from chromatic contrast, stimuli to measure chromatic contrast are isoluminant (equal in luminance) and vary only in chromaticity. To quantify chromatic con- trast, differences between chromaticities ("inten- sities of color") need to be calculated.

\section{Contrast Indices}

Contrast indices calculate differences in chroma- ticities relative to a reference intensity, which is supposed to correspond to the adaptation of the observer. Mainly three indices of contrast have been used.

The Weber contrast is calculated by the dif- ference in intensity between two colors divided by the intensity of the background. This index is sensible if the background corresponds to the observer's adaptation color, and the perception of the difference depends on the intensity of this background.

Alternatively, the Michelson contrast divides the difference between intensities by the sum of their intensities. This approach is sensible when it may be assumed that observers are adapted to the intensities of the two colors, rather than to the background. Both indices are equivalent when the two colors vary symmetrically around the background. These two indices are useful for specifying contrast in sine-wave gratings, for example, when measuring contrast sensitivity (e.g., $[4,7]$ ).

Finally, the root mean square or RMS contrast consists in the standard deviation of the 
chromatic differences across a scene. In order to relativize these differences to the overall mean, the values are standardized before the calculation of the RMS index. This index may not only be used for contrast sensitivity measure- ments but also for contrast distributions in scenes. Apart from these three main indices, still other indices to assess contrast are possible [1].

\section{Color Differences}

As with color in general, the quantification of chromatic contrast is relative to perception. Color does not directly map to a physical mea- sure. Consequently, metrics of chromatic contrast are relative to the dimensions used to quantify chromaticity ("intensity of color"). For the measurement of contrast sensitivity, two ways to quantify chromatic contrast have been used.

First, contrast may be determined by the physical or instrument contrast [2]. The instrument contrast is the relative intensity of two chromatic lights (component colors), such as two monitor primaries or two monochromatic lights (e.g., [7]). The contrast between the two unmixed component colors corresponds to $100 \%$, and they are mixed to produce intermediate levels of contrast (cf. Michelson contrast). The physical intensity is typically measured as luminance. However, the component colors, the dimension along which the difference is measured, and the scaling of the differences are arbitrary and depend on the devise used to produce the colors.

Second, cone contrast is used as a perceptual measure of contrast [1,8]. Cone contrasts are calculated by the difference in absorption

(or excitation) of each of the three photoreceptors (L-, M-, and S-cones) relative to the state of adaptation of that photoreceptor. Weber or Michelson contrast may be used for this pur- pose. Alternatively, colors may be directly represented in cone contrast space, in which each of the three dimensions (DLMS) reflects the cone contrast for one type of cone. The RMS contrast may be used in order to combine the contrasts of the three cones.

Note, that perceptual quantifications depend on the perceptual processes that are modeled by the difference metric and on the knowledge about these processes. Cone contrasts relate chromatic contrast sensitivity to the low-level, cone- opponent processes of color vision. Hence, com- parisons in contrast across different chromatic- ities are relative to these mechanisms. Higher- level, cortical mechanisms might involve differ- ent kinds or reweighed contrasts, and hence quan- tifications of contrast might be different for highlevel mechanisms. Moreover, studies may differ in how they define maximum cone contrast, which affects the relative scaling of the cone- opponentaxes.

Spatial Frequency

In order to control spatial frequencies, gratings that alternate periodically between the 
contrasting colors are used. Sharp edges may occur between the single bars of the grating and between the grating and the background. Such sharp edges imply high spatial frequency compo- nents, which need to be separated from the spatial frequency component determined by the bars of the gratings.

To avoid such edges between the bars, grat- ings are sinusoidally modulated. To further avoid sharp edges between grating and background, contrasts are gradually reduced toward the edges through the application of a filter. As a result, the grating smoothly melts into the back- ground, and the strongest stimulation (i.e., con- trast) is at the center of the grating. The molding of the overall grating through a filter is called an envelope, and in most cases this filter consists in a Gaussian function (Gaussian envelope).

A sinusoidally modulated grating with an enve- lope is called a Gabor patch (e.g., [10]).

While contrast may be controlled as the ampli- tude of the sinusoids, spatial frequency corre- sponds to the frequency of cycles. Since the perceived spatial frequency depends on the reti- nal image, it also depends on the distance of the eye to the grating. For this reason, spatial fre- quencies are quantified as cycles per degree (cpd) of visual angle because the visual angle is independent of the distance. Stimuli should be large enough to show a sufficient number of cycles for all frequencies (e.g., 2.5 cycles, cf. [1]).

For gratings above 3-4 cpd, chromatic aberra- tion produces luminance artifacts that influence detection thresholds. This is mainly the case for blue-yellow gratings, since the wavelength com- positions for red and green chromaticities do not differ strongly, resulting in a similar refraction $[7,14]$.

In order to measure chromatic contrast sensi- tivity, gratings may be presented, for example, at different locations of the display or with different orientations, and the observer has to indicate the location and the orientation of the grating, respec- tively, through a forced-choice response.

\section{Temporal Frequency}

High temporal frequencies correspond to fast flicker between contrasting chromaticities, low frequencies to slow, and gradual transitions. Temporal frequency is quantified as cycles per second, that is, Hertz (Hz).

Temporal contrast sensitivity is measured through heterochromatic flicker. In this setup, chromaticities periodically change, and temporal transitions are sinusoidally modulated to control single temporal frequencies. At high frequencies, chromaticities are seen as an unchanging mixture of the contrasting chromaticities (flicker fusion). At extremely low frequencies, the change in chromaticity is not visible. For example, the slow change in chromaticity of daylight remains usually unnoticed in everyday life. Note that achromatic artifacts occur in L-M flicker due to latency differences between L- and M-cones 
[1].

Spatiotemporal contrast sensitivity, i.e., con- trasts sensitivity as a function of spatial and temporal frequency, may be measured by oscil- lating between gratings that are phaseshifted by 90- and hence show spatially inverse (green-red- green vs. red-green-red) contrasts [4].

\section{Chromatic Contrast Sensitivity Functions}

The relationship between contrast sensitivity and spatial and temporal frequency is captured through chromatic contrast sensitivity functions (cCSF). In CSFs, contrast sensitivity is represented along the y-axis as a function of spatial frequency (spatial contrast sensitivity function (sCSF)) or temporal frequency (temporal contrast sensitivity function (tCSF)). All axes are usually scaled logarithmically.

Spatial Contrast Sensitivity Functions

Chromatic spatial contrast sensitivity functions are low-pass [7]. This means that contrast sensitivity declines with high spatial frequencies ("narrower lines") but barely declines when spa- tial frequency approaches zero. The chromatic sensitivity function reaches its maximum close to zero cpd, where the change between contrasting colors is completely gradual.

This pattern contrasts the one found for ach- romatic sCSFs. Achromatic sCSFs are bandpass with a peak at about 3-5 cpd and decreasing sensitivity toward both higher and lower spatial frequencies. In fact, at low spatial frequency $(<0.5 \mathrm{cpd})$, chromatic contrast sensitivity is higher than achromatic contrast sensitivity when measured as cone contrasts. This is the case when the transition between contrasted colors covers the whole fovea ( 2-, i.e., a thumb at arm's length). At the same time, acuity is much higher for achromatic (cutoff at 40-60 cpd) than for L-M (about $12 \mathrm{cpd}$ ) and S-(L+M) contrasts (10 cpd). In fact, due to chromatic aberration, the effective resolution is only $3-4 \mathrm{cpd}$ for the $\mathrm{S}-(\mathrm{L}+\mathrm{M})$ contrast.

Moreover, increasing eccentricity from the fovea toward the periphery has a stronger attenu- ating effect on L-M contrast sensitivity than on achromatic contrast sensitivity, while $\mathrm{S}-(\mathrm{L}+\mathrm{M})$

contrast sensitivity declines with eccentricity in a similar way as achromatic contrast sensitivity $[1,8]$

In sum, "very thin" stripes are still visible when shown in black and white but "disappear" when shown as isoluminant colors [7]. This has been used, for example, in image compression (e.g., TV broadcast) where high spatial frequency information is saved in achromatic contrast only and chromatic high spatial frequency information is discarded 
[13].

Temporal Contrast Sensitivity Functions

Temporal frequency modulates chromatic con- trast sensitivity in a similar way as spatial fre- quency $[1,5]$. It is mainly low-pass, with only a slight attenuation in sensitivity below about $1 \mathrm{cpd}$. It is highest when colors change one to three times per second $(1-3 \mathrm{~Hz})$, and temporal resolution is at about $30-40 \mathrm{~Hz}$. In contrast, ach- romatic contrast sensitivity peaks between 5 and $20 \mathrm{~Hz}$ and has a higher cutoff value for temporal resolution $(\sim 50$ $\mathrm{Hz}$ ). Since both, spatial and tem- poral cCSFs are low-pass, the spatiotemporal cCSF is also low-pass [4].

Due to the lower temporal resolution for differences in chromaticity, heterochromatic flicker can be used to exactly determine isoluminance for an individual observer (heterochromatic flicker photometry). At a temporal frequency of $15-20 \mathrm{~Hz}$, chromaticities of contrasting colors fuse, and the only flicker left is achromatic. The brightness of one of the contrasting colors is adjusted until there is no flicker, which implies that the luminance of the two colors is equal.

Development

As with adults, the spatial (L-M) CSF is low-pass in infancy (8-30 weeks) and develops through a steady increase in overall sensitivity and in spa- tial resolution [12]. In contrast, temporal (L-M) CSFs are band-pass in 3-month-old infants and develop an adultlike lowpass profile only at the age of 4 months [2]. Across the life-span, chro- matic contrast sensitivity increases steadily until adolescence and decreases thereafter [6].

\section{Eye Movements}

While sensitivity for luminance contrast decreases during smooth pursuit, sensitivity for isoluminant $\mathrm{L}-\mathrm{M}$ and $\mathrm{S}-(\mathrm{L}+\mathrm{M})$ contrast increases (10-16\%), starting about $50 \mathrm{~ms}$ before eye movement [10]. An increase in contrast sensitiv- ity has also been found during optokinetic nys- tagmus, but not vestibulo-ocular reflex [11]

\section{Theories on Chromatic Contrast Sensitivity}

Physiological Basis

The optics of the eye blur the retinal image and explain a major part of sensitivity loss for high spatial frequencies for both chromatic and achro- matic contrasts. Blurring through chromatic aber- ration further reduces $\mathrm{S}-(\mathrm{L}+\mathrm{M})$ contrasts of high spatial frequency in the retinal image. Moreover, the distribution of cones across the retina (cone mosaic) also modulates contrast sensitivity. In particular, the scarcity of S-cones in the retina further attenuates the spatial resolution of $\mathrm{S}-(\mathrm{L}+\mathrm{M})$ contrast sensitivity [14]. 
Chromatic contrasts are processed by two independent pathways that go from the retinal ganglion cells via the lateral geniculate nucleus (LGN) to the visual cortex. The parvocellular pathway processes L-M, the koniocellular path- way $\mathrm{S}-(\mathrm{L}+\mathrm{M})$ contrasts. The decrease in contrast sensitivity with eccentricity agrees with the dis- tribution of parvocellular and koniocellular receptive fields across the retina [1, 8]. Moreover, the increase of chromatic contrast sensitivity dur- ing pursuit and optokinetic nystagmus indicates a boost of the parvo- and koniocellular system.

\section{Functional Role in Ecology}

Chromatic contrasts are statistically independent from luminance contrast in natural scenes and hence provide an additional source of information for object identification apart from luminance contrast [3]. Furthermore, spatial L-M contrast sensitivity is optimal for the detection of red and yellow fruits at reaching distance [9].

\section{References}

1. Diez-Ajenjo, M.A., Capilla, P.: Spatio-temporal con- trast sensitivity in the cardinal directions of the colour space. A review. J. Optom. 3(1), 2-19 (2010)

2. Dobkins, K.R., Anderson, C.M., Lia, B.: Infant tem- poral contrast sensitivity functions (tCSFs) mature earlier for luminance than for chromatic stimuli: evi- dence for precocious magnocellular development? Vision Res. 39(19), 3223-3239 (1999)

3. Hansen, T., Gegenfurtner, K.R.: Independence of color and luminance edges in natural scenes. Vis. Neurosci. 26(1), 35-49 (2009). doi:10.1017/ S0952523808080796. S0952523808080796 [pii]

4. Kelly, D.H.: Spatiotemporal variation of chromatic and achromatic contrast thresholds. J. Opt. Soc. Am. 73(6), 742-750 (1983)

5. 5. Kelly, D.H., van Norren, D.: Two-band model of heterochromatic flicker. J. Opt. Soc. Am. 67(8), 1081-1091 (1977)

6. 6. Knoblauch, K., Vital-Durand, F., Barbur, J.L.: Varia- tion of chromatic sensitivity across the life span. Vision Res. 41(1), 23-36 (2001)

7. 7. Mullen, K.T.: The contrast sensitivity of human col- our vision to red-green and blue-yellow chromatic gratings. J. Physiol. 359, 381-400 (1985)

8. 8. Mullen, K.T., Kingdom, F.A.: Differential distribu- tions of red-green and blue-yellow cone opponency across the visual field. Vis. Neurosci. 19(1), 109-118 (2002)

9. 9. Parraga, C.A., Troscianko, T., Tolhurst, D.J.: Spatiochromatic properties of natural images and human vision. Curr. Biol. 12(6), 483-487 (2002). doi: S0960982202007182 [pii]

10. 10. Sch€utz,A.C.,Braun,D.I.,Kerzel,D.,Gegenfurtner,K. R.: Improved visual sensitivity during smooth pursuit eye movements. Nat. Neurosci. 11(10), 1211-1216 (2008). doi:10.1038/nn.2194. nn.2194 [pii]

11. 11. Sch€utz, A.C., Braun, D.I., Gegenfurtner, K.R.: Chro- matic contrast sensitivity during optokinetic nystag- mus, visually enhanced vestibulo-ocular reflex, and smooth pursuit eye movements. J. Neurophysiol. 101(5), 2317-2327 (2009). doi:10.1152/ jn.91248.2008

12. 12. Teller, D.Y.: Spatial and temporal aspects of infant color vision. Vision Res. 38(21), 3275-3282 (1998)

13. 13. Watson, A.B..: Perceptual-components architecture for digital video. J. Opt. Soc. Am. A Opt. 
Image Sci. 7(10), 1943-1954 (1990)

14. 14. Williams, D., Sekiguchi, N., Brainard, D.: Color, contrast sensitivity, and the cone mosaic. Proc. Natl. Acad. Sci. U. S. A. 90(21), 9770-9777 (1993) 